\title{
BMJ Open Intra-articular botulinum toxin A for base-of-thumb osteoarthritis: protocol for a randomised trial (RHIBOT)
}

Charlotte Gil, ${ }^{1}$ Hendy Abdoul, ${ }^{2}$ Raphaël Campagna, ${ }^{3}$ Henri Guerini, ${ }^{3}$ Estelle leong, ${ }^{1}$ Franck Chagny, ${ }^{1}$ Catherine Bedin, ${ }^{1}$ Alexandra Roren, ${ }^{1,4,5}$

Marie-Martine Lefèvre-Colau, ${ }^{1,4,5,6}$ Serge Poiraudeau, ${ }^{1,4,5,6}$ Antoine Feydy, François Rannou, ${ }^{1,6,7}$ Christelle Nguyen ${ }^{1,6,7}$

To cite: Gil C, Abdoul H, Campagna $\mathrm{R}$, et al. Intraarticular botulinum toxin $\mathrm{A}$ for base-of-thumb osteoarthritis: protocol for a randomised trial (RHIBOT). BMJ Open 2018;8:e022337. doi:10.1136/ bmjopen-2018-022337

- Prepublication history and additional material for this paper are available online. To view these files, please visit the journal online (http://dx.doi org/10.1136/bmjopen-2018022337).

Received 12 February 2018 Revised 26 April 2018 Accepted 4 June 2018
Check for updates

For numbered affiliations see end of article.

Correspondence to Dr Christelle Nguyen; christelle.nguyen2@aphp.fr

\section{ABSTRACT}

Introduction Recent studies have suggested that intra-articular injection of botulinum toxin A (BTA) may have analgesic effects in degenerative joint diseases. We aim to assess the efficacy of intra-articular injection of BTA associated with splinting in patients with trapeziometacarpal osteoarthritis (TMC OA).

Methods and analysis We will conduct a randomised double-blind controlled trial. Overall, 60 individuals with TMC OA fulfilling adapted 1990 American College of Rheumatology criteria for hand $O A$ will be recruited in one tertiary care centre in France and randomised to receive splinting + a single ultrasound-guided injection in the TMC joint of 50 Allergan Units of BTA resuspended in $1 \mathrm{~mL}$ saline or splinting $+1 \mathrm{~mL}$ saline. Randomisation will be centralised. The allocation ratio will be $1: 1$. The primary outcome will be the mean change from baseline in base-of-thumb pain on a self-administered 11-point Numeric Rating Scale in 10-point increments at 3 months after injection. Secondary outcomes will be the mean change in base-of-thumb pain at 1 and 6 months, mean change in hand-specific activity limitations assessed by the self-administered Cochin Hand Function Scale, proportion of responders assessed by the Osteoarthritis Research Society International -Outcome Measures in Rheumatology (OMERACT) criteria and consumption of analgesics and non-steroidal antiinflammatory drugs assessed by a self-administered 4-class scale at 3 and 6 months. Cointerventions will be allowed in both groups and will be self-reported. Adverse events will be recorded at 3 and 6 months. Participants, care providers and statisticians will be blinded to the allocated treatment.

Ethics and dissemination The RHIBOT trial has been authorised by the Agence Nationale de Sécurité du Médicament and approved by the Comité de Protection des Personnes de Tours Ouest-1. The findings of the study will be disseminated in peer-reviewed journals and at conferences. If the results are positive, intra-articular BTA could be an efficient and safe complementary therapeutic option for patients with TMC $0 A$.

Date and version identifier of the protocol 8 January 2018, V. 2.0.

Trial registration number NCT03187626; Pre-results.

\section{Strengths and limitations of this study}

- First randomised controlled trial to assess the effects of intra-articular botulinum toxin $A$ on pain for people with painful base-of-thumb osteoarthritis.

- A trial conducted independently from industrial funding.

- Outcomes selected in accordance with 0steoarthritis Research Society International and European Society on Clinical and Economic Aspects of Osteoporosis, Osteoarthritis and Musculoskeletal Diseases recommendations for clinical trials in osteoarthritis.

- A comparator chosen to warrant allocation concealment and to minimise biases associated with the absence of participant blinding in order to specifically assess the treatment effect of intra-articular botulinum toxin $\mathrm{A}$.

- Participants will be recruited from a tertiary care centre and may not be representative of the whole French population with base-of-thumb osteoarthritis.

\section{INTRODUCTION}

Trapeziometacarpal osteoarthritis (TMC OA) is a common condition affecting middle-aged and older people. ${ }^{1}$ TMC OA induces pain and closure of the first web, which in turn causes altered thumb-index pinch and, therefore, limitations in hand-specific activities. ${ }^{1}$ In 2009 , in a randomised multicentre controlled trial of 112 participants (101 women) with TMC OA, our group showed that a custommade night-time splint $(n=57)$ had positive effects on pain and hand function at 1 year as compared with usual care $(n=55) .^{2}$ Other reviews have shown the positive effect on pain of splinting in TMC OA. ${ }^{3} 4$ However, for short-term and mid-term effects, guidelines are inconsistent. Therapeutic options usually include splinting, exercise therapy and intra-articular treatments. The European League Against Rheumatism recommends intra-articular injections of glucocorticoids and considers injections of hyaluronic acid 
useful, ${ }^{5}$ but no specific recommendations have been formulated by the American College of Rheumatology. ${ }^{6}$

Recently, the use of intra-articular botulinum toxin A (BTA) as a pain modulator in various types of musculoskeletal conditions has raised intense interest. BTA is a potent neurotoxin produced by the bacterium Clostridium botulinum. BTA inhibits acetylcholine release into the synaptic cleft in cholinergic nerve terminals, causing muscle paralysis. In humans, BTA was originally used for its muscle paralysing effects in neuromuscular disorders. Preclinical experimental studies conducted in dogs (OA) ${ }^{78}$ horses (synovitis) ${ }^{9}$ or mice ${ }^{10} 11$ with various painful joint conditions showed an intrinsic antinociceptive effect of intra-articular BTA. ${ }^{12}$

The exact mechanisms of pain modulation by BTA in $\mathrm{OA}$ are unclear. Pain in OA involves both nociceptive and neuropathic complex mechanisms, and abnormal excitability in peripheral and central pain pathways. ${ }^{13} 14$ BTA could suppress the secretion of some neurotransmitters, thus directly decreasing peripheral sensitisation and indirectly decreasing central sensitisation. ${ }^{15}$ BTA could also have an inhibitory role on the release of mediators involved in nociception, such as substance $\mathrm{P}$, calcitonin gene-related peptide and glutamate.

Trials assessing the clinical effect of intra-articular BTA in knee OA suggest a mid-term positive effect on pain ${ }^{16}$ and no marked toxicity, ${ }^{17}$ but the overall level of evidence is low. Only one study assessing the effects of intra-articular BTA in eight participants with TMC OA was registered but was stopped early for lack of funding and slow accrual (ClinicalTrials.gov Identifier: NCT01045694).

We hypothesised that a single ultrasound-guided intra-articular injection of BTA associated with splinting in people with painful TMC OA may reduce pain at 3 months. Our study aims at assessing the 'add-on' effect of an intra-articular injection of BTA to splinting, rather than assessing the effect of an intra-articular injection of BTA as a stand-alone treatment. Therefore, we will offer splinting in both groups.

\section{METHODS AND ANALYSIS}

\section{Design overview}

This is a prospective, two parallel-group, double-blind, randomised, placebo-controlled, single-centred study. Duration of follow-up for each participant will be 6 months after intra-articular injection. The study will be reported in accordance with the Consolidated Standards of Reporting Trials statement and its relevant extensions for non-pharmacological trials and abstracts. ${ }^{18}$ The intervention will be reported in accordance with the Template for Intervention Description and Replication checklist and guide. ${ }^{19}$

\section{Setting and participants}

The recruitment will be prospective, among inpatients and outpatients of the rehabilitation department of a tertiary care centre in France (Cochin Hospital, Paris) consulting a physician or referred to an occupational therapist for TMC OA, by advertisement through a specialised network of specialists and occupational therapists, articles posted on the department's website, posters placed in the investigating centre and information leaflets given to patients during consultations. People willing to participate in the study will be invited to contact a management centre by phone or email. A biomedical research technician will preliminarily screen people for eligibility criteria, then, if appropriate, schedule a face-to-face baseline visit with one of the investigators, a senior specialist in physical and rehabilitation medicine with previous experience as a trialist and/or who received dedicated training to enrol participants in the RHIBOT trial by the coordinating investigator. In addition, patients whose computerised medical record mentions the diagnosis of TMC OA between 2015 and 2017 will be contacted by the biomedical research technician by phone or mail and invited to participate in the study, if appropriate.

The main eligibility criteria will be age 18 years and older, pain intensity on a self-administered 11-point Numeric Rating Scale (NRS) $\geq 30$ (0, no pain, to 100 , maximal pain, in 10-point increments), pain involving the base of thumb, X-ray evidence of TMC OA with at least two of the four following items involving the TMC joint: osteophytes, joint space narrowing, subchondral bone sclerosis or subchondral cysts and 1990 American College of Rheumatology classification criteria for hand $\mathrm{OA}$ adapted to TMC OA. ${ }^{20} \mathrm{~A}$ full description of the inclusion and non-inclusion criteria is in online supplementary appendix 1 . People excluded for temporary reasons can be rescreened.

\section{Experimental group}

Participants allocated to the experimental group will receive a custom-made thermoformable plastic splint to be worn for 48 hours after a single ultrasound-guided injection of 50 Allergan Units of BTA (Botox, Allergan) resuspended in $1 \mathrm{~mL}$ saline in the TMC joint, then nightly for 6 months after the intra-articular injection.

On the same day as the randomisation, the thermoformable plastic splint will be custom made by one of the three trained occupational therapists of the Rehabilitation Department of Cochin Hospital in accordance with a standardised procedure. Briefly, the splint will be a rigid rest orthosis made of thermoformable plastic as previously described. ${ }^{2}$ It will cover the base of the thumb and the thenar eminence. Occupational therapists will adjust the splint for each patient so that the first web will remain open and the thumb will be in opposition with the first long finger (online supplementary appendix 2). Participants will be recommended to contact the occupational therapists if they feel that the splint needs adjustment (eg, pain increased while wearing the splint or adverse effects such as skin erosion). They will be instructed to wear the splint continuously for 48 hours after the intra-articular injection, then only at night, as much as possible for 6 months. No patient will switch groups once randomised. 
Compliance with wearing the splint will be self-reported at 3 and 6 months after injection and recorded in the electronic case report form. No specific measures will be taken to enhance compliance with the splint.

On the same day as randomisation, participants will receive a single ultrasound-guided injection of 50 Allergan Units of BTA resuspended in $1 \mathrm{~mL}$ saline in the TMC joint. The pharmacist will prefill a syringe containing the product to inject according to the randomisation assignment and hand-deliver the numbered treatment box containing the prefilled syringe to the radiology department. Two board-certified radiologists (RC, HG), with expertise in administering intra-articular injections, with experience in scoring ultrasound in patients with hand OA, will perform the injections in a blinded and standardised manner in the Radiology B Department of Cochin Hospital. The intra-articular injection will be performed while the patient is supine to avoid discomfort and malaise; with the hand lying palm up on the table, through the thenar muscle, with the transducer parallel to the axis of the needle to visualise the latter's advancement to the palmar recess of the TMC joint. US images will be obtained with an Aplio500 scanner using a multifrequency linear array 12-18.0 MHz PLT-1204BX transducer (Toshiba Medical Systems, Tokyo, Japan) focused at the level of TMC joint. Ultrasound scoring for synovitis and osteophytes will be performed in a standardised manner, using a prespecified checklist, as previously described. ${ }^{21}$ BTA will be injected in the TMC joint using a $25 \mathrm{G}$ and $25 \mathrm{~mm}$ long needle under ultrasound control (12-18 MHz linear array transducer) and strict aseptic conditions. Correct needle tip positioning will be visually assessed by tracing air in the joint. ${ }^{22}$ Immediately after the intervention, the success of blinding will be assessed by the credibility/expectancy questionnaire..$^{23} 24$

\section{Control group}

On the same day as randomisation, participants allocated to the control group will receive a custom-made thermoformable plastic splint to be worn for 48 hours after a single ultrasound-guided injection of $1 \mathrm{~mL}$ of saline in the TMC joint, then nightly for 6 months after the intra-articular injection, following the same procedure as for the experimental group. Because of no gold standard for intra-articular treatment in patients with TMC OA, we chose saline as the comparator because of its innocuity and its identical volume, colour, viscosity and echogenicity as BTA, which allows for maintaining blinding of radiologists and participants. Indeed, among sources of biases in OA trials, only patient blinding was recently found to have an impact on the results. ${ }^{25}$ Hyaluronic acid was discussed as a comparator but was eventually discarded because it has not shown beneficial clinical effects over other intra-articular treatments in patients with TMC OA ${ }^{26}$; its viscosity is different from BTA, which might confer efficacy in itself and interfere with the blinding process and the cost of $1 \mathrm{~mL}$ hyaluronic acid is 2500 -fold that of $1 \mathrm{~mL}$ saline in France.

\section{Cointerventions}

Pharmacological and non-pharmacological treatments usually prescribed for TMC OA will be authorised. Analgesics and oral and topical non-steroidal anti-inflammatory drugs, intra-articular injections (hyaluronic acid and corticosteroids), symptomatic slow-acting drugs for OA, physiotherapy and home-based therapeutic exercises will be self-reported by patients by use of self-administered standardised checklists, then recorded in the electronic case report form by the biomedical research technician.

\section{Outcomes}

Primary and secondary efficacy outcomes were selected in accordance with the Osteoarthritis Research Society International (OARSI) recommendations ${ }^{27}$ and the guidelines for the conduct of pharmacological clinical trials in hand OA of the European Society on Clinical and Economic Aspects of Osteoporosis, Osteoarthritis and Musculoskeletal Diseases (ESCEO).$^{28}$ The primary efficacy endpoint will be the mean change from baseline in mean BT pain intensity in the previous 48 hours on a self-administered 11-point NRS (0, no pain, to 100 , maximal pain, in 10-point increments) at 3 months after injection. This timepoint is the one recommended by the ESCEO for fast-acting drugs. ${ }^{28}$ Secondary efficacy endpoints will be the mean change from baseline in mean base-of-thumb pain intensity in the previous 48 hours on a self-administered 11-point NRS ( 0 , no pain, to 100 , maximal pain, in 10-point increments) at 1 and 6 months; mean change from baseline in mean hand-specific limitations in activities in the previous 2 weeks on the self-administered Cochin Hand Function Scale (CHFS; 0, no limitations, to 90 , maximal limitations) $)^{29}{ }^{30}$ at 3 and 6 months; mean change from baseline in patient global assessment on a self-administered 11-point NRS (0, worst possible, to 100 , best possible, in 10-point increments) at 3 and 6 months; percentage of OARSI responders (OARSI response is defined as an improvement in pain (0-100 NRS) or function (0-90 CHFS) $\geq 50 \%$ and absolute change $\geq 20 / 100$ on pain NRS or $\geq 9 / 90$ on CHFS, or improvement in at least two of the three following items: (1) pain $\geq 20 \%$ and absolute change $\geq 10 / 100$ on pain NRS, (2) function $\geq 20 \%$ and absolute change $\geq 9 / 90$ on CHFS, (3) patient global assessment $\geq 20 \%$ and absolute change $\geq 10 / 100$ on patient global assessment NRS) ${ }^{31}$ at 3 and 6 months; and the self-reported consumption of analgesics (non-opioid, weak and strong opioids) and non-steroidal anti-inflammatory drugs on a self-administered 4-class scale (never; several times a month; several times a week; daily) at 3 and 6 months. We did not plan any scoring of X-rays neither at baseline nor during follow-up because we do not expect our treatment to have a structural effect in the short term. For participants who discontinue or deviate from intervention protocols, the same outcome data will be collected if possible.

Safety outcome will be recorded by the investigator by asking an open-ended question ('Have you had any adverse events since last contact?') during a face-to-face 
visit at 3 months and by mail, email or phone at 6 months after the intra-articular injection. If the participant missed the appointment, a biomedical research technician will request information on adverse events by mail, email or phone and record them. All adverse events will be recorded in the 'adverse events' section of the electronic case report form by the investigator. The investigator will assess the causality relation between the adverse event and the clinical trial. The method will be based on WHO-Uppsala Monitoring Centre method and will include the following four causality terms: (1) certain, (2) probable/ likely, (3) possible and (4) unlikely (not excluded). ${ }^{32}$

\section{Randomisation and allocation concealment}

All individuals who meet the inclusion criteria and agree to participate will be randomised during the inclusion visit by the investigator by use of a randomisation list computer generated by an independent statistician from the Unité de Recherche Clinique. The allocation ratio will be 1:1. Randomisation will be centralised by a coordinating team from the Unité de Recherche Clinique Cochin Necker, who is not involved in the enrolment, follow-up or assessment of participants. The list will have variable block sizes. Only the independent statistician from the Unité de Recherche Clinique and the informatician from the coordinating team will have access to the randomisation list. The allocation will be concealed by a computer-generated interface in the electronic case report form (CleanWeb software).

Participants, investigators, occupational therapists, radiologists, statisticians and treating physicians will be blinded to the allocation group. The pharmacist who will prepare the solution to inject in accordance with the randomisation list will have participants' anonymised code for each solution prepared. The pharmacist will have no contact with the participants or the investigators and will work independently of the study team. Injected solutions for both experimental and control groups will have the same volume, colour, viscosity and echogenicity. Treatment administration and clinical monitoring of the experimental products will be the same in the experimental and control groups. If necessary, blinding can be broken by the Délégation à la Recherche Clinique et à l'Innovation or antipoison centre of Fernand-Widal Hospital, Paris, France. All information about breaking the blind, such as reason, time and justification will be noted in the electronic case report form.

\section{Statistical aspects}

The sample size is estimated at 60 patients. We predicted a difference in mean change from baseline of 15 points on the pain NRS between the experimental and control groups, with an SD of 20 points, and a power (1- $\beta$ ) of $80 \%$, corresponding to 29 patients in each arm. Considering $3.3 \%$ lost to follow-up, we will need to enrol an estimated 30 patients for each arm. Fifteen points on the pain NRS is considered the minimal clinically perceived difference in pain for patients with hand OA. ${ }^{33}$
All statistical analyses will be performed in accordance with a prespecified statistical analysis plan by an independent senior biostatician from the Unité de Recherche Clinique Cochin Necker, who will be blinded to the allocation group and who will have no contact with the participants. All analyses will be performed on an intention-to-treat basis, in that all patients will be considered in the analysis and will be analysed in the group to which they had been assigned. There will be no interim analyses. Participants lost to follow-up will not be replaced. In case of missing data on the primary endpoint criterion, baseline carried forward imputation will be carried out.

For descriptive analyses, categorical variables will be reported with absolute numbers and percentages and quantitative variables with mean (SD) or median (IQR). For comparative analyses, categorical data will be analysed by Fisher's exact test or $\mathrm{X}^{2}$ test and quantitative data by Student's t-test or Wilcoxon signed-rank test with an alpha risk of $5 \%$ and statistical significance level $p=0.05$. The difference in the change between baseline and postbaseline values will be assessed by analysis of covariance or constrained longitudinal data analysis. Logistic regression models may be used to explore response to treatment by using OARSI criteria. We did not prespecify any subgroup analyses stratified for the severity of structural damage because our sample size will be too small to ensure statistical soundness. The statistical analysis will be further detailed in a dedicated statistical analysis plan before any analysis is undertaken.

\section{Patient and public involvement}

Patients and/or public were not involved.

\section{Participant timeline}

\section{Baseline visit}

The biomedical research technician will set up a baseline face-to-face visit with one of the investigators after a preliminary screening of eligibility criteria by phone. During this visit, the investigator will again check inclusion and exclusion criteria, give oral and written information about the study and collect informed oral and written consent from the patient. Patients who are eligible and agree to participate will be included and randomised on the same day. Baseline characteristics (age, sex, body mass index, educational level, professional status, family history of TMC OA, dominant hand, symptom duration, date of last X-ray, Kapandji score for opposition and counter-opposition of the painful thumb) ${ }^{34}$ will be recorded by the investigator in the electronic case report form. Participants will complete printed versions of the self-administered questionnaires prespecified in the protocol. The answers will be recorded in the electronic case report form by the biomedical research technician. Participants will receive a personal card indicating their participation in the study, their identification number and the contact details of the investigator, the managing centre and the antipoison centre. Female participants of childbearing age will be recommended to have an efficient 
Table 1 Schedule of enrolment, interventions and assessments

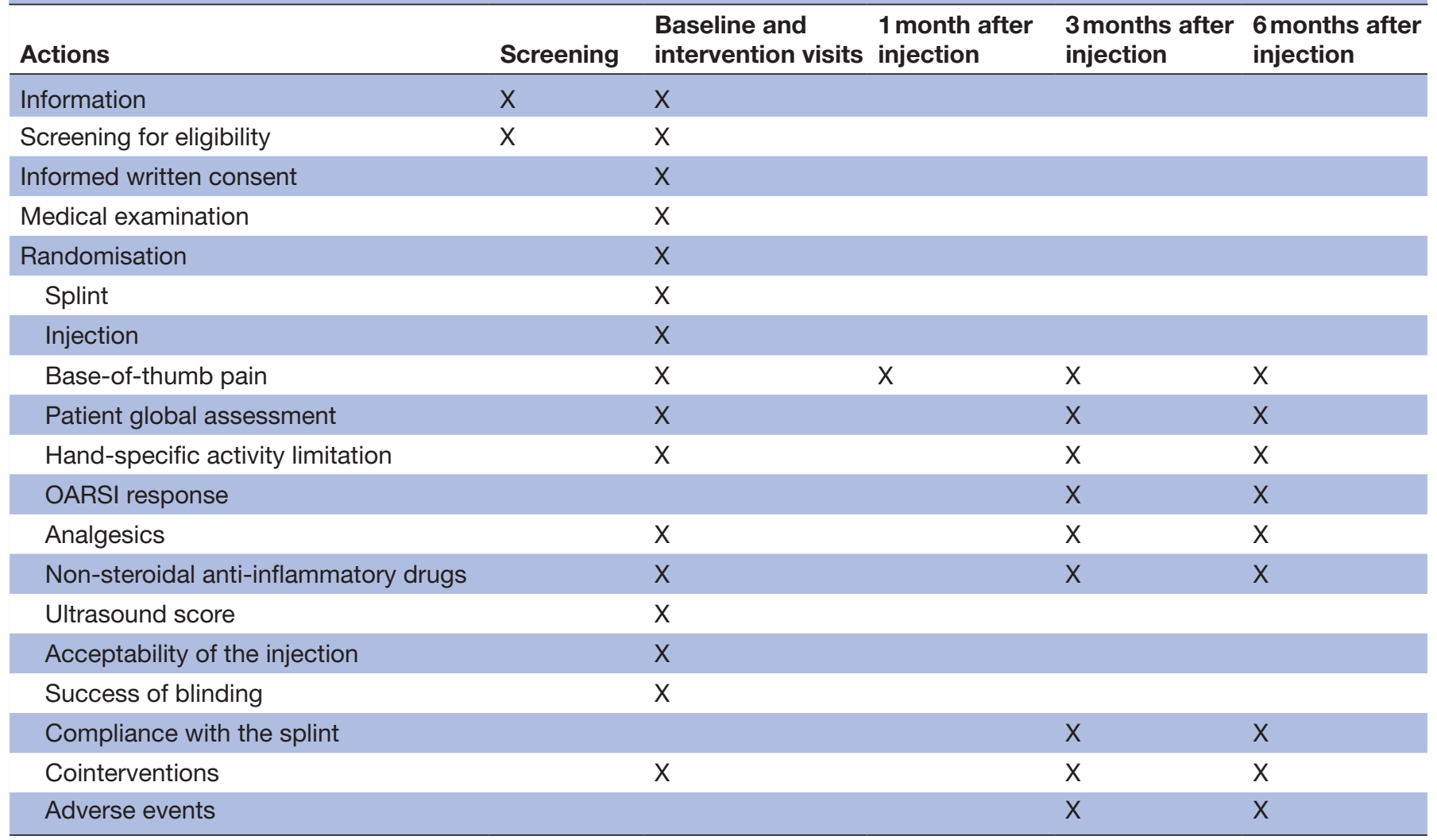

OARSI, Osteoarthritis Research Society International.

contraceptive method during the whole study. Indeed, clinical guidelines for the use of BTA advise against injection during pregnancy because there is no clear data to evaluate a clinical malformative risk or fetotoxicity. This was also a request from our institutional review board (Comité de Protection des Personnes de Tours Ouest-1) (table 1).

On the same day as the baseline visit and the randomisation Participants will receive a thermoformable plastic splint custom made as described previously and will receive a single ultrasound-guided injection of 50 Allergan Units of BTA resuspended in $1 \mathrm{~mL}$ saline (experimental group) or $1 \mathrm{~mL}$ saline (control group) in the TMC joint with one of the two senior radiologists in accordance with the allocated treatment and the procedure described previously. Participants will complete printed versions of the self-administered questionnaires before injection and the credibility/expectancy questionnaire to assess the success of blinding after injection. The answers will be recorded in the electronic case report form by the biomedical research technician. Radiologists will record the side injected, grades of synovitis and osteophytes illustrated by screen shots and the correct needle tip positioning by tracing air in the palmar recess. Radiologists will remind participants to wear the thermoformable plastic splint continuously for 48 hours after the intra-articular injection, then only at night, as much as possible for 6 months.

\section{Three-month visit}

The investigator will assess participants during a faceto-face visit at 3 months after injection. In the case the patient missed the 3-month follow-up visit, outcomes will be assessed by phone, email or mail by a clinical research technician. Prespecified efficacy outcomes will be collected by using printed self-administered questionnaires. Safety outcomes, compliance with the splint and cointerventions will be self-reported by patients by using standardised self-administered checklists, and then recorded in the electronic case report form by a biomedical research technician. If the participant missed the appointment, a biomedical research technician will collect the information by mail, email or phone and record it in the electronic case report form.

\section{One-month and 6-month follow-up}

Participants will be assessed by mail, email or telephone at 1 and 6 months after the intra-articular injection. Prespecified efficacy outcomes will be collected by using self-administered questionnaires. Safety outcomes, compliance with wearing the splint and cointerventions will be self-reported by patients by using standardised self-administered checklists, and then will be recorded in the electronic case report form by a biomedical research technician.

End of the research

At end of the research, participants will continue their usual medical follow-up. No exclusion period for any other research is required. 


\section{Data management}

All data will be securely stored at the Unité de Recherche Clinique. They will be anonymised by a predefined code, given to every patient included, according to a defined protocol. Data will be recorded in a secured electronic case report form completed by the investigators and biomedical research technicians. The forms will be protected by a personal access code. A clinical research associate will be responsible for the safety and protection of the participants, the accuracy of the information and the compliance with the predefined protocol. The clinical research associate can conduct an audit trail at any stage of the project. The clinical research associate will have access to source data through the investigator, at any time of the study, as legally required (articles L.1121-3 and R.5121-13 of the French Code de Santé Publique). The coordinating investigator signed a responsibility engagement. In accordance with the French Good Clinical Practices, the sponsor, Assistance Publique-Hôpitaux de Paris, will be responsible for obtaining the consent and permission of all parties involved in the research and ensuring proper coordination between the location of the trial, source data and documents, quality control and audit if necessary. The sponsor will not be directly involved in the study or in the writing of the publication or decision to publish.

\section{ETHICS AND DISSEMINATION}

\section{Ethical considerations}

Methods for obtaining consent from the research participants and information given

According to French Law (Article L.1122-1-1 of the French Code de Santé Publique), free and informed consent from the participant is required for inclusion in a research protocol. Participants will receive information at least 7 days before they give oral and written informed consent during the baseline face-to-face inclusion visit, which is recorded in the medical file. The original dated and signed document will be kept in the file. A copy of the consent signed by the participant and the investigator will be given to the participant, as will an information leaflet about the study.

\section{Data confidentiality}

Confidentiality will be guaranteed by use of a personal code for each participant, predefined at inclusion. Each professional working for this study is under medical secret oath (according to articles 226-13 and 226-14 of the French Penal Code). The final data will be accessible only to investigators, statisticians and biomedical research technicians. There will be no contractual agreement that will limit access to the final trial dataset.

\section{Legal obligations}

Assistance Publique-Hôpitaux de Paris is the sponsor of this research and by delegation, the Département de la Recherche Clinique et du Développement (Hopital
Saint-Louis, 1 avenue Claude Vellefaux, 75010 Paris) performs the research missions in accordance with Article L.1121-1 of the French Code de Santé Publique. Assistance Publique-Hôpitaux de Paris obtained authorisation from the Agence Nationale de Sécurité du Médicament on 7 August 2017 (No 2017/46PP), the Commission Nationale de l'Informatique et des Libertés on 13 October 2017 (No 2101482v0) and the Comité de protection des personnes de Tours Ouest-1 on 19 December 2017 (No 2017T1-26).

\section{Modifications to the research}

In case of modifications to the research, approval by the Assistance Publique-Hôpitaux de Paris, Comité de protection des personnes de Tours Ouest-1 and Agence Nationale de Sécurité du Médicament is required. The information leaflet and consent form can be modified if needed.

\section{Research committee}

The scientific committee will include senior specialists in physical and rehabilitation medicine $(\mathrm{CN}, \mathrm{FR})$ and radiologists (AF, RC, HG), with previous experience as a trialist, and a methodologist (HA), whose mission will be to define the objectives of the study, write the protocol and submit modifications to the protocol during the trial, with annual meetings. The steering committee will include $\mathrm{CN}, \mathrm{FR}, \mathrm{HA}$ and $\mathrm{AB}$ (see acknowledgements), whose mission will be to define the general organisation of the study, coordinate information, determine methodology, monitor it and propose changes during the study. The Délégation à la Recherche Clinique et à l'Innovation as the promotor of the study will have final decision on modifications.

\section{Safety considerations}

Any adverse events during the study must be assessed by the investigator, to evaluate their seriousness. Adverse events must be documented as best possible by the investigator in case of a serious adverse event, and the intensity of the event must be graded: mild, moderate or severe. The investigator must evaluate a possible link of causality with the drug tested, on a four-point scale: certain, probable/likely, possible or unlikely. ${ }^{32}$ In case of a serious adverse event, the investigator will immediately notify the promotor, in accordance with article R.1123-49 of the French Code de Santé Publique. The promotor will assess the seriousness of the adverse event, its causality link with the drug and the expected or unexpected characteristic. The investigator will notify the sponsor, immediately on the day when he/ she becomes aware of any serious adverse event, except for events that are mentioned in the informed consent form (muscle weakness, difficulty swallowing, pneumonia due to a false food route or fluid in the respiratory tract and water and/or sodium retention (hypernatraemia)).

\section{Dissemination plan}

We aim to publish the findings of RHIBOT in a peer-reviewed scientific journal and diffuse them to physical 
and rehabilitation medicine physicians at national and international conferences. The authors will be responsible for all articles or press releases regarding the results, with no help from professional writers. No results will be communicated before the end of the study. Assistance Publique-Hôpitaux de Paris will be the owner of the data. The original protocol and the full dataset will be available by contacting the coordinating investigator, CN (christelle.nguyen2@aphp.fr). RHIBOT is the first randomised controlled trial to assess the efficacy of BTA in patients with TMC OA. If the results are positive, intra-articular BTA could be an efficient and safe complementary therapeutic option for patients with TMC OA.

\section{Author affiliations}

${ }^{1}$ AP-HP, Hôpitaux Universitaires Paris Centre-Groupe Hospitalier Cochin, Service de Rééducation et de Réadaptation de l'Appareil Locomoteur et des Pathologies du Rachis, Paris, France

${ }^{2}$ Unité de Recherche Clinique - Centre d'Investigation Clinique Paris Descartes Necker/Cochin, Hôpital Tarnier, Paris, France

${ }^{3}$ AP-HP, Hôpitaux Universitaires Paris Centre-Groupe Hospitalier Cochin, Service de Radiologie B, Paris, France

${ }^{4}$ INSERM UMR 1153, Centre de Recherche Épidémiologie et Statistique, Sorbonne Paris Cité, ECaMO Team, Paris, France

${ }^{5}$ Institut Fédératif de Recherche sur le Handicap, Paris, France

${ }^{6}$ Faculté de Médecine, Sorbonne Paris Cité, Université Paris Descartes, Paris, France ${ }^{7}$ INSERM UMR 1124, Laboratoire de Pharmacologie, Toxicologie et Signalisation Cellulaire, Faculté des Sciences Fondamentales et Biomédicales, Centre Universitaire des Saints-Pères, Paris, France

Acknowledgements The authors thank Alexandra Bruneau from URC-CIC Paris Descartes Necker/Cochin for implementation, monitoring, and data management and Laura Smales for professional copyediting.

Contributors Conception and design of the study: $\mathrm{HA}, \mathrm{RC}, \mathrm{HG}, \mathrm{El}, \mathrm{FC}, \mathrm{CB}, \mathrm{AR}$, $A F, M-M L-C, S P, F R$ and CN. Drafting of the original protocol: RC, SP, FR and CN. Coordination of the study: FR and CN. Design of the statistical analysis plan: HA and $\mathrm{CN}$. Drafting of the present manuscript: $C G$ and $C N$. Obtaining of funding: $C N$. Final approval: CG, HA, RC, AF, HG, El, FC, CB, AR, AF, M-ML-C, FR and CN.

Funding This work was supported by the Assistance Publique-Hôpitaux de Paris, Contrat de Recherche Clinique 2016 (CRC16125).

Competing interests None declared.

Patient consent Obtained.

Ethics approval Comité de Protection des Personnes Tours Ouest-I.

Provenance and peer review Not commissioned; externally peer reviewed.

Open access This is an open access article distributed in accordance with the Creative Commons Attribution Non Commercial (CC BY-NC 4.0) license, which permits others to distribute, remix, adapt, build upon this work non-commercially, and license their derivative works on different terms, provided the original work is properly cited and the use is non-commercial. See: http://creativecommons.org/ licenses/by-nc/4.0/

(C) Article author(s) (or their employer(s) unless otherwise stated in the text of the article) 2018. All rights reserved. No commercial use is permitted unless otherwise expressly granted.

\section{REFERENCES}

1. Poole JU, Pellegrini VD. Arthritis of the thumb basal joint complex. $J$ Hand Ther 2000;13:91-107.

2. Rannou F, Dimet J, Boutron I, et al. Splint for base-of-thumb osteoarthritis: a randomized trial. Ann Intern Med 2009;150:661-9.

3. Spaans AJ, van Minnen LP, Kon M, et al. Conservative treatment of thumb base osteoarthritis: a systematic review. J Hand Surg Am 2015;40:16-21.

4. Bertozzi L, Valdes K, Vanti C, et al. Investigation of the effect of conservative interventions in thumb carpometacarpal osteoarthritis: systematic review and meta-analysis. Disabil Rehabil 2015;37:2025-43.

5. Zhang W, Doherty M, Leeb BF, et al. EULAR evidence based recommendations for the management of hand osteoarthritis: report of a Task Force of the EULAR Standing Committee for International Clinical Studies Including Therapeutics (ESCISIT). Ann Rheum Dis 2007;66:377-88.

6. Hochberg MC, Altman RD, April KT, et al. American College of Rheumatology 2012 recommendations for the use of nonpharmacologic and pharmacologic therapies in osteoarthritis of the hand, hip, and knee. Arthritis Care Res 2012;64:465-74.

7. Hadley HS, Wheeler JL, Petersen SW. Effects of intra-articular botulinum toxin type A (Botox) in dogs with chronic osteoarthritis. Vet Comp Orthop Traumatol 2010;23:254-8.

8. Heikkilä HM, Hielm-Björkman AK, Morelius M, et al. Intra-articular botulinum toxin A for the treatment of osteoarthritic joint pain in dogs: a randomized, double-blinded, placebo-controlled clinical trial. Vet J 2014;200:162-9.

9. DePuy T, Howard R, Keegan K, et al. Effects of intra-articular botulinum toxin type $\mathrm{A}$ in an equine model of acute synovitis: a pilot study. Am J Phys Med Rehabil 2007;86:777-83.

10. Anderson S, Krug H, Dorman C, et al. Analgesic effects of intraarticular botulinum toxin Type $B$ in a murine model of chronic degenerative knee arthritis pain. J Pain Res 2010;3:161-8.

11. Mahowald ML, Krug HE, Singh JA, et al. Intra-articular Botulinum Toxin Type A: a new approach to treat arthritis joint pain. Toxicon 2009;54:658-67.

12. Malfait AM, Schnitzer TJ. Towards a mechanism-based approach to pain management in osteoarthritis. Nat Rev Rheumatol 2013;9:654-64.

13. Perrot S. Osteoarthritis pain. Best Pract Res Clin Rheumatol 2015;29:90-7.

14. Schaible HG. Mechanisms of chronic pain in osteoarthritis. Curr Rheumatol Rep 2012;14:549-56.

15. Nguyen C, Lefèvre-Colau MM, Poiraudeau S, et al. Evidence and recommendations for use of intra-articular injections for knee osteoarthritis. Ann Phys Rehabil Med 2016;59:184-9.

16. Singh JA. Use of botulinum toxin in musculoskeletal pain. F1000Res 2013;2:52.

17. Nguyen C, Rannou F. The safety of intra-articular injections for the treatment of knee osteoarthritis: a critical narrative review. Expert Opin Drug Saf 2017;16:897-902.

18. Boutron I, Altman DG, Moher D, et al. CONSORT statement for randomized trials of nonpharmacologic treatments: a 2017 update and a CONSORT extension for nonpharmacologic trial abstracts. Ann Intern Med 2017;167:40-7.

19. Hoffmann TC, Glasziou PP, Boutron I, et al. Better reporting of interventions: template for intervention description and replication (TIDieR) checklist and guide. BMJ 2014;348:g1687.

20. Altman R, Alarcón G, Appelrouth D, et al. The American College of Rheumatology criteria for the classification and reporting of osteoarthritis of the hand. Arthritis Rheum 1990;33:1601-10.

21. Keen HI, Lavie F, Wakefield RJ, et al. The development of a preliminary ultrasonographic scoring system for features of hand osteoarthritis. Ann Rheum Dis 2008;67:651-5.

22. Qvistgaard E, Kristoffersen H, Terslev L, et al. Guidance by ultrasound of intra-articular injections in the knee and hip joints. Osteoarthritis Cartilage 2001;9:512-7.

23. Borkovec TD, Nau SD. Credibility of analogue therapy rationales. J Behav Ther Exp Psychiatry 1972;3:257-60.

24. Devilly GJ, Borkovec TD. Psychometric properties of the credibility/expectancy questionnaire. J Behav Ther Exp Psychiatry 2000;31:73-86.

25. Bolvig J, Juhl CB, Boutron I, et al. Some Cochrane risk-ofbias items are not important in osteoarthritis trials: a metaepidemiological study based on Cochrane reviews. J Clin Epidemiol 2018;95.

26. Trellu S, Dadoun S, Berenbaum F, et al. Intra-articular injections in thumb osteoarthritis: A systematic review and meta-analysis of randomized controlled trials. Joint Bone Spine 2015;82:315-9.

27. McAlindon TE, Driban JB, Henrotin Y, et al. OARSI Clinical Trials Recommendations: Design, conduct, and reporting of clinical trials for knee osteoarthritis. Osteoarthritis Cartilage 2015;23:747-60.

28. Reginster JL, Arden NK, Haugen IK, et al. Guidelines for the conduct of pharmacological clinical trials in hand osteoarthritis: Consensus of a Working Group of the European Society on Clinical and Economic Aspects of Osteoporosis, Osteoarthritis and Musculoskeletal Diseases (ESCEO). Semin Arthritis Rheum 2017.

29. Duruöz MT, Poiraudeau S, Fermanian J, et al. Development and validation of a rheumatoid hand functional disability scale that assesses functional handicap. J Rheumatol 1996;23:1167-72. 
30. Poiraudeau S, Chevalier X, Conrozier T, et al. Reliability, validity, and sensitivity to change of the Cochin hand functional disability scale in hand osteoarthritis. Osteoarthritis Cartilage 2001;9:570-7.

31. Pham T, van der Heijde D, Altman RD, et al. OMERACT-OARSI initiative: Osteoarthritis Research Society International set of responder criteria for osteoarthritis clinical trials revisited. Osteoarthritis Cartilage 2004;12:389-99.

32. WHO-umc.org. Standardised case causality assessment, 2012.

33. Tubach F, Ravaud P, Martin-Mola E, et al. Minimum clinically important improvement and patient acceptable symptom state in pain and function in rheumatoid arthritis, ankylosing spondylitis, chronic back pain, hand osteoarthritis, and hip and knee osteoarthritis: Results from a prospective multinational study. Arthritis Care Res 2012;64:1699-707.

34. Kapandji A. [Clinical test of apposition and counter-apposition of the thumb]. Ann Chir Main 1986;5:67-73.

35. Lefevre-Colau MM, Poiraudeau S, Oberlin C, et al. Reliability, validity, and responsiveness of the modified Kapandji index for assessment of functional mobility of the rheumatoid hand. Arch Phys Med Rehabil 2003;84:1032-8. 